\title{
Impact of intracranial pressure monitoring on mortality in patients with traumatic brain injury: a systematic review and meta-analysis
}

\author{
${ }^{*}$ Qiang Yuan, MD, Xing Wu, MD, PhD, Yirui Sun, MD, PhD, Jian Yu, MD, PhD, Zhiqi Li, MD, \\ Zhuoying Du, MD, PhD, Ying Mao, MD, PhD, Liangfu Zhou, MD, PhD, and Jin Hu, MD, PhD \\ Department of Neurosurgery, Huashan Hospital, Fudan University, Shanghai, PR China
}

OBJECT Some studies have demonstrated that intracranial pressure (ICP) monitoring reduces the mortality of traumatic brain injury (TBI). But other studies have shown that ICP monitoring is associated with increased mortality. Thus, the authors performed a meta-analysis of studies comparing ICP monitoring with no ICP monitoring in patients who have suffered a TBI to determine if differences exist between these strategies with respect to mortality, intensive care unit (ICU) length of stay (LOS), and hospital LOS.

METHODS The authors systematically searched MEDLINE, EMBASE, and the Cochrane Central Register of Controlled Trials (Central) from their inception to October 2013 for relevant studies. Randomized clinical trials and prospective cohort, retrospective observational cohort, and case-control studies that compared ICP monitoring with no ICP monitoring for the treatment of TBI were included in the analysis. Studies included had to report at least one point of mortality in an ICP monitoring group and a no-ICP monitoring group. Data were extracted for study characteristics, patient demographics, baseline characteristics, treatment details, and study outcomes.

RESULTS A total of 14 studies including 24,792 patients were analyzed. The meta-analysis provides no evidence that ICP monitoring decreased the risk of death (pooled OR 0.93 [95\% $\mathrm{Cl} 0.77-1.11$ ], $\mathrm{p}=0.40$ ). However, 7 of the studies including 12,944 patients were published after 2012 (January 2012 to October 2013), and they revealed that ICP monitoring was significantly associated with a greater decrease in mortality than no ICP monitoring (pooled OR $0.56[95 \% \mathrm{Cl}$ $0.41-0.78], p=0.0006$ ). In addition, 7 of the studies conducted in North America showed no evidence that ICP monitoring decreased the risk of death, similar to the studies conducted in other regions. ICU LOSs were significantly longer for the group subjected to ICP monitoring (mean difference [MD] 0.29 [95\% $\mathrm{Cl} 0.21-0.37] ; p<0.00001$ ). In the pooled data, the hospital LOS with ICP monitoring was also significantly longer than with no ICP monitoring (MD $0.21[95 \% \mathrm{Cl}$ $0.04-0.37] ; p=0.01$ ).

CONCLUSIONS In this systematic review and meta-analysis of ICP monitoring studies, the authors found that the current clinical evidence does not indicate that ICP monitoring overall is significantly superior to no ICP monitoring in terms of the mortality of TBI patients. However, studies published after 2012 indicated a lower mortality in patients who underwent ICP monitoring.

http://thejns.org/doi/abs/10.3171/2014.10.JNS1460

KEY WORDS intracranial pressure monitoring; mortality; outcome; traumatic brain injury; meta-analysis

$\mathrm{T}$ RaUmatic brain injury (TBI) is a common cause of death and disability worldwide. ${ }^{17}$ Despite some claims to the contrary, no clear decrease in severe TBI-related mortality or improvement in overall outcome has been observed over the past 2 decades. ${ }^{24}$
Recently, many studies have shown that the presence and frequency of high intracranial pressure (ICP) are predictive of the outcome of severe TBI. ${ }^{18,23}$ Thus, ICP monitoring has been widely used in the management of severe TBI. Indeed, the Brain Trauma Foundation (BTF) guide-

\footnotetext{
ABBREVIATIONS BTF = Brain Trauma Foundation; GCS = Glasgow Coma Scale; ICP = intracranial pressure; ICU = intensive care unit; MD = mean difference; STROBE = Strengthening the Reporting of Observational Studies in Epidemiology; TBI = traumatic brain injury. SUBMITTED January 9, 2014. ACCEPTED October 22, 2014.

INCLUDE WHEN CITING Published online December 5, 2014; DOI: 10.3171/2014.10.JNS1460.

DISCLOSURE The authors report no conflict of interest concerning the materials or methods used in this study or the findings specified in this paper. This work was supported by the National Natural Science Foundation of China (NSFC Grants 30371454, 81271375, and 81171133), the Science and Technology Commission of Shanghai Municipality Project (10JC1402300), and Shanghai Nature Science Foundation (08411952000).

* Drs. Qiang Yuan, Xing Wu, and Yirui Sun contributed equally to this work.
} 
lines recommend ICP monitoring in all patients who have suffered severe TBI (Glasgow Coma Scale [GCS] score < 9) with a CT scan revealing intracranial pathology (Level II recommendation) or in severe TBI patients with a normal CT scan but with 2 or more of the following risk factors: age older than 40 years, unilateral or bilateral motor posturing, or systolic blood pressure lower than $90 \mathrm{~mm}$ $\mathrm{Hg}$ (Level III recommendation). ${ }^{4,6}$ Despite these recommendations, significant variability remains in the practice of ICP monitoring. ${ }^{5,19,22,26,29}$ This may reflect conflicting or absent clinical evidence regarding the benefit of ICP monitoring on outcomes in TBI patients.

Although a trial of ICP monitoring in patients having suffered TBI (a large multicenter randomized study) was published in 2012, controversy remains due to issues with the design of that trial. ${ }^{7}$ That study included only a subset of patients in South America, where the use of ICP monitoring is generally low. ${ }^{25}$

Although a Cochrane review on the topic was published recently, no study was analyzed because only randomized trials were included. ${ }^{9}$ Without considering observational studies, the review was unable to provide any conclusion as to the value of ICP monitoring in patients with TBI. Thus, we conducted a meta-analysis of ICP monitoring studies, including observational studies, to evaluate the evidence of a relationship between the use of ICP monitoring and outcome in patients with TBI.

\section{Methods}

\section{Data Sources and Searches}

We searched MEDLINE, EMBASE, and the Cochrane Central Register of Controlled Trials (Central) from their inception to October 2013 for relevant studies using appropriate combinations of MeSH terms and key words, including intracranial pressure, intracranial hypertension, ICP monitor, intracranial pressure monitoring, TBI, traumatic brain injury, brain injury, and craniocerebral trauma. We performed this relatively wide search to include the maximum number of relevant patients.

\section{Study Selection}

Randomized clinical trials and prospective cohort, retrospective observational cohort, and case-control studies that compared ICP monitoring with no ICP monitoring for the treatment of TBI were included in our analysis. Studies that did not include patients who did not undergo ICP monitoring as the comparator were excluded. Also excluded were articles not containing original research (e.g., narrative reviews, editorials, and case reports). Studies included had to report at least one point of mortality in an ICP monitoring group and a no-ICP monitoring group.

\section{Data Abstraction}

According to preestablished eligibility criteria, 2 reviewers (Q.Y. and X.W.) independently reviewed all citations and selected eligible studies using a standardized data abstraction form. Disagreements were resolved by consensus. Data were extracted for 1) study characteristics (author and year of publication, country of origin, study design, and sample size), 2) patient demograph- ics (age and sex) and baseline characteristics (severity of injury), 3) treatment details (ICP monitoring or not, type of ICP monitor used, and duration of ICP monitoring), and 4) study outcomes (mortality, intensive care unit [ICU] length of stay [LOS], and hospital LOS in different groups). In instances of duplicate reporting, we used data from the study that included the largest number of patients or, when available, individual patient data from each study. We contacted authors for clarification on study samples or for missing data.

\section{Methodological Quality of Included Studies}

We used the criteria for reporting observational studies proposed in the STROBE (Strengthening the Reporting of Observational Studies in Epidemiology) statement to complete a methodological evaluation of the included observational studies. ${ }^{32}$

\section{Statistical Analysis}

Most statistical analyses were performed using the Review Manager Software (version 5.2, Cochrane Collaboration). All tests were 2-sided, and the significance level was set at 0.05. Statistical analyses for continuous variables or dichotomous variables were conducted. Mean differences (MDs) were used for the analysis of continuous variables (ICU LOS and hospital LOS), and odds ratios were used for dichotomous variables (mortality). The distribution of ICU LOS and hospital LOS were right-skewed and we thus log-transformed them to yield a normal distribution. ${ }^{3}$ A log-normal distribution facilitated the analysis and presentation of outcomes between groups. The association between ICP monitoring and TBI mortality was expressed as odds ratios with $95 \%$ confidence intervals. If the value "1" was excluded from the 95\% confidence interval, the point estimate of the odds ratios was considered to have reached statistical significance $(\mathrm{p}<0.05)$. The generic inverse variance method was used to analyze the odds ratio. The I-squared $\left(\mathrm{I}^{2}\right)$ statistic was used to measure the extent of inconsistency among the results. ${ }^{15}$ Heterogeneity was detected using the chi-square test. Because the chi-square test lacks power when the number of studies is small, we considered significant heterogeneity to be present when both the chisquare value was within the $10 \%$ level of significance (p $<0.10)$ and the $\mathrm{I}^{2}$ value exceeded $50 \%$.

In cases of heterogeneity, summary measures of the effect of ICP monitoring for mortality were obtained by conducting a random-effects meta-analysis of the besteffect estimate available from each study, which assumes that studies were a random sample of a hypothetical population of studies and assigns a weight to each study, taking into account variance within and between studies. When an adjusted odds ratio was available from the paper, it was taken as the best estimate; otherwise, the crude estimate was used. The extent to which the combined risk estimate might be affected by individual studies was assessed by consecutively omitting each study from the meta-analysis. Subgroup analysis considering more homogeneous sets of studies was used as an additional sensitivity test. Publication bias (linked to the fact that negative trials are 
cited less frequently and are therefore more likely to be missed in the search for relevant studies) was assessed using the "funnel plot" technique, based on a graph plotting effect estimates against sample size. Additionally, Egger regression and the Begg and Mazumdar methods were used to evaluate publication bias using Stata software (version 10, Stata Corp.). Multiple meta-regression was performed using Stata 10 to identify the factors causing the heterogeneity.

\section{Quality of Evidence}

Studies were graded and assigned a quality rating with respect to the key question according to the CEBM (Centre for Evidence-Based Medicine) criteria. Studies were graded from Level 1 (strongest evidence, such as reports with clinical decision rules and high-quality validating studies) to Level 5 (weakest evidence, often expert opinion). ${ }^{14}$

\section{Results \\ Study Selection}

In total, 7175 studies were identified and screened for retrieval using the strategy described above. After screening the title and abstract, 7047 studies were excluded and 128 were retrieved and subjected to detailed evaluations. Based on the inclusion and exclusion criteria, 114 of those studies were excluded, and thus 14 studies were included in the meta-analysis (Fig. 1). All included studies were published, peer-reviewed papers.

\section{Study Characteristics}

Thirteen studies were observational cohort studies and 1 was a randomized controlled trial. Tables 1 and 2 describe the 14 eligible studies according to their design. Nine studies conducted a multivariate analysis with an adjusted odds ratio of mortality in the ICP monitoring group compared with the no-ICP monitoring group. The randomized controlled trial was conducted in South America. Of the observational cohort studies, 7 were conducted in North America (US or Canada), 2 in Austria, 2 in China, 1 in Saudi Arabia, and 1 in the Netherlands. Nine studies contained information on in-hospital mortality, 4 on ICU mortality, 3 on 6-month mortality, 2 on 2-week mortality, and 1 study contained information on 28-day mortality. Six studies contained enough information on ICU LOS to be included in the meta-analysis, and 4 contained information on hospital LOS.

\section{Methodological Quality of Studies Included}

In the 13 observational cohort studies, 4 presented a flow diagram for participants, 13 studies adequately described their study population (including missing data and patients lost during follow-up), and 5 presented their funding sources. Table 3 provides a more complete evaluation of methodological quality.

\section{Outcome Measures}

The studies were clearly heterogeneous $\left(\mathrm{I}^{2}=82 \%, \mathrm{p}<\right.$ $0.001)$. Heterogeneity was not eliminated by considering only observational cohort studies, only studies reporting in-hospital mortality, or only studies including severe TBI patients $\left(\mathrm{I}^{2}=83 \%\right.$, df $12, \mathrm{p}<0.001 ; \mathrm{I}^{2}=84 \%$, df $8, \mathrm{p}<$ 0.001 , and $\mathrm{I}^{2}=82 \%$, df $10, \mathrm{p}<0.001$, respectively). Because of this heterogeneity, a random-effects model was used.

Fourteen studies compared mortality due to TBI in the ICP monitoring group and the no-ICP monitoring group, with ORs ranging from 0.15 to 2.80 . The meta-analysis provides no evidence that ICP monitoring decreased the risk of death (pooled OR 0.93 [95\% CI 0.77-1.11], p = 0.40 ). After excluding the randomized controlled trial, the 13 observational cohort studies evaluated the association between ICP monitoring and mortality in TBI patients and also showed no evidence for decreased risk of death with ICP monitoring (pooled OR 0.95 [95\% CI 0.79-1.15], $\mathrm{p}=$ 0.61; Fig. 2).

However, 7 of the studies including 12,944 patients were published after 2012 (January 2012 to October 2013). These studies revealed that ICP monitoring was significantly associated with a greater decrease in mortality than no ICP monitoring (pooled OR 0.56 [95\% CI 0.41-0.78], $\mathrm{p}=0.0006$; Fig. 3), although the other 7 studies, published before 2012, showed that ICP monitoring was not significantly associated with a greater decrease in mortality (pooled OR 1.15 [95\% CI 0.94-1.40], p = 0.17; Fig. 3). In addition, 7 of the studies conducted in North America showed no evidence that ICP monitoring decreased the risk of death, similar to the studies conducted in other regions (Fig. 4).

Regarding the 11 studies in which only severe TBI patients (GCS score < 9) were evaluated, a meta-analysis revealed that ICP monitoring was not significantly associated with a greater decrease in mortality than no ICP monitoring at the end of follow-up (pooled OR 0.91 [95\% CI 0.71-1.17], $p=0.47$; Fig. 5). The following subgroup analysis showed that ICP monitoring was significantly associated with a greater decrease in mortality than no ICP monitoring in the studies published after 2012 (pooled OR 0.59 [95\% CI 0.42-0.81], $p=0.001$; Fig. 5), but was not associated with a greater decrease in those published before 2012 (pooled OR 1.29 [95\% CI 0.93-1.79], p = 0.13; Fig. 5).

Nine of the studies including 22,313 patients reported data on in-hospital mortality at the end of follow-up and revealed that ICP monitoring did not decrease the risk of in-hospital mortality (pooled OR 1.06 [95\% CI 0.80-1.42], $\mathrm{p}=0.68$ ). Four studies contained information on ICU mortality, and 3 studies contained information on 6-month mortality. Meta-analyses showed that ICP monitoring was not significantly associated with a greater decrease in either ICU or 6-month mortality. However, only 2 studies contained information on 2-week mortality; ICP monitoring did decrease the risk of 2-week mortality (pooled OR 0.63 [95\% CI 0.45-0.88], $\mathrm{p}=0.007$; Fig. 6).

Six of the studies including 3309 patients in the metaanalysis reported data on ICU LOS. ICU LOSs were significantly longer for the group subjected to ICP monitoring (MD 0.29 [95\% CI 0.21-0.37], p < 0.00001; Fig. 7). Four of the studies including 2604 patients in the metaanalysis reported data on hospital LOS. In the pooled data, the hospital LOS with ICP monitoring was significantly longer than with no ICP monitoring (MD 0.21 [95\% CI 0.04-0.37], $p=0.01$; Fig. 8). 


\section{Relevant articles identified and} screened for retrieval $(n=7175)$

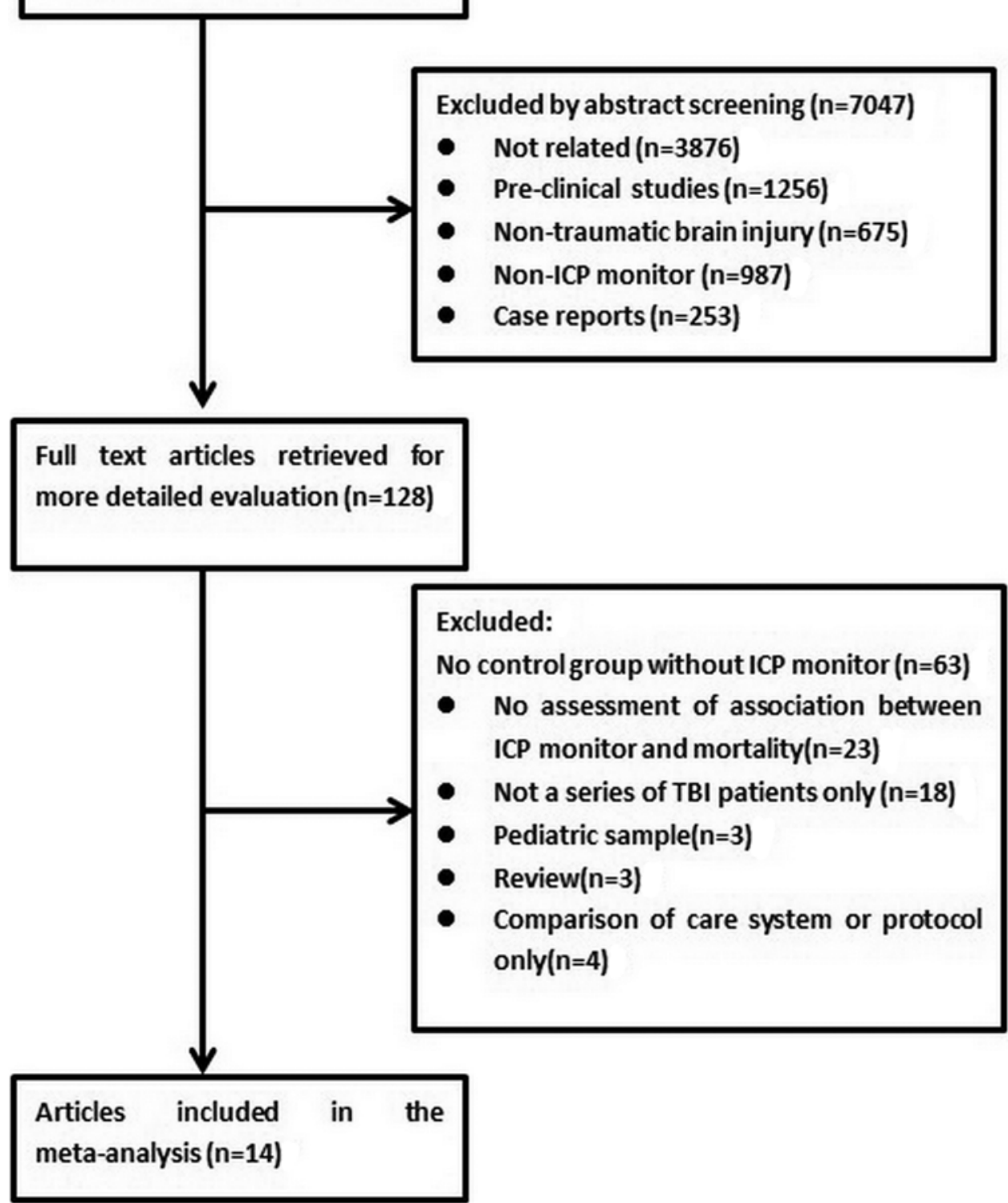

FIG. 1. Identification process for eligible studies. Of the 7175 studies initially identified from our electronic search, 14 met the inclusion criteria and were included in this meta-analysis.

To investigate potential sources of heterogeneity, several analyses were performed. The leave-one-out procedure revealed that no single study was responsible for the heterogeneity (heterogeneity tests were always significant when one study at a time was excluded).

In performing the meta-regression, we considered the following covariates: year of publication, country of origin, sample size, severity of TBI, and type of mortality. Meta-regression showed that only the year of publication was significantly associated with OR estimates: in particular, publication after 2012 correlated with lower OR (slope point estimate: $0.689 ; \mathrm{p}=0.037$ ).

\section{Publication Bias}

Visual evaluations of funnel plots indicated no publication bias (Figs. 9-12). Also, no publication bias was detected by Egger regression (bias 0.433, SE 0.712, p = 0.554 ), or by Begg and Mazumdar rank correlation (Kendall's $\mathrm{z}=-0.27, \mathrm{p}=0.784$ ).

\section{Discussion}

To our knowledge, this is the first reported meta-analysis to evaluate the association between ICP monitoring and mortality in patients with TBI. Stein et al. conducted 


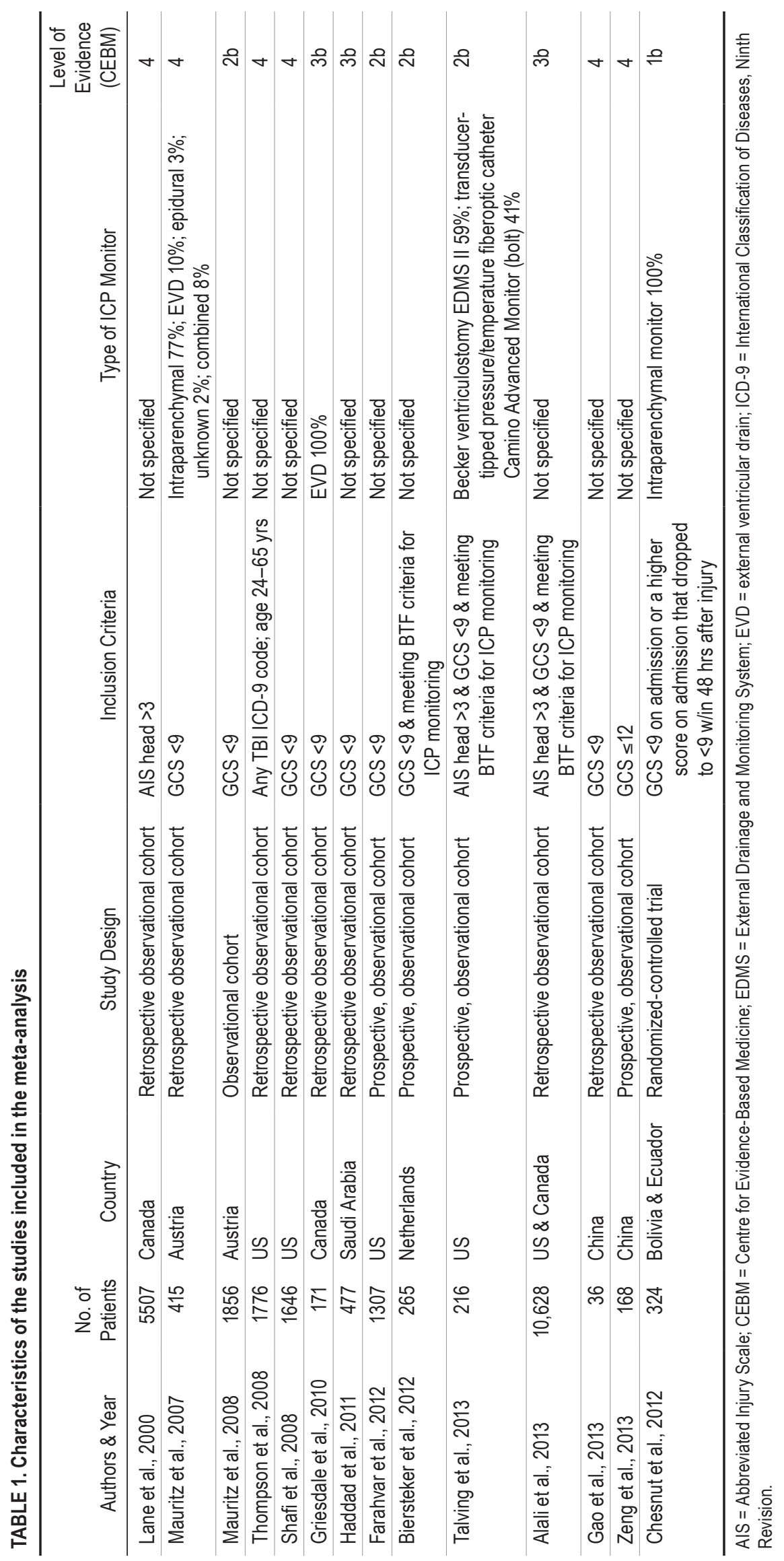




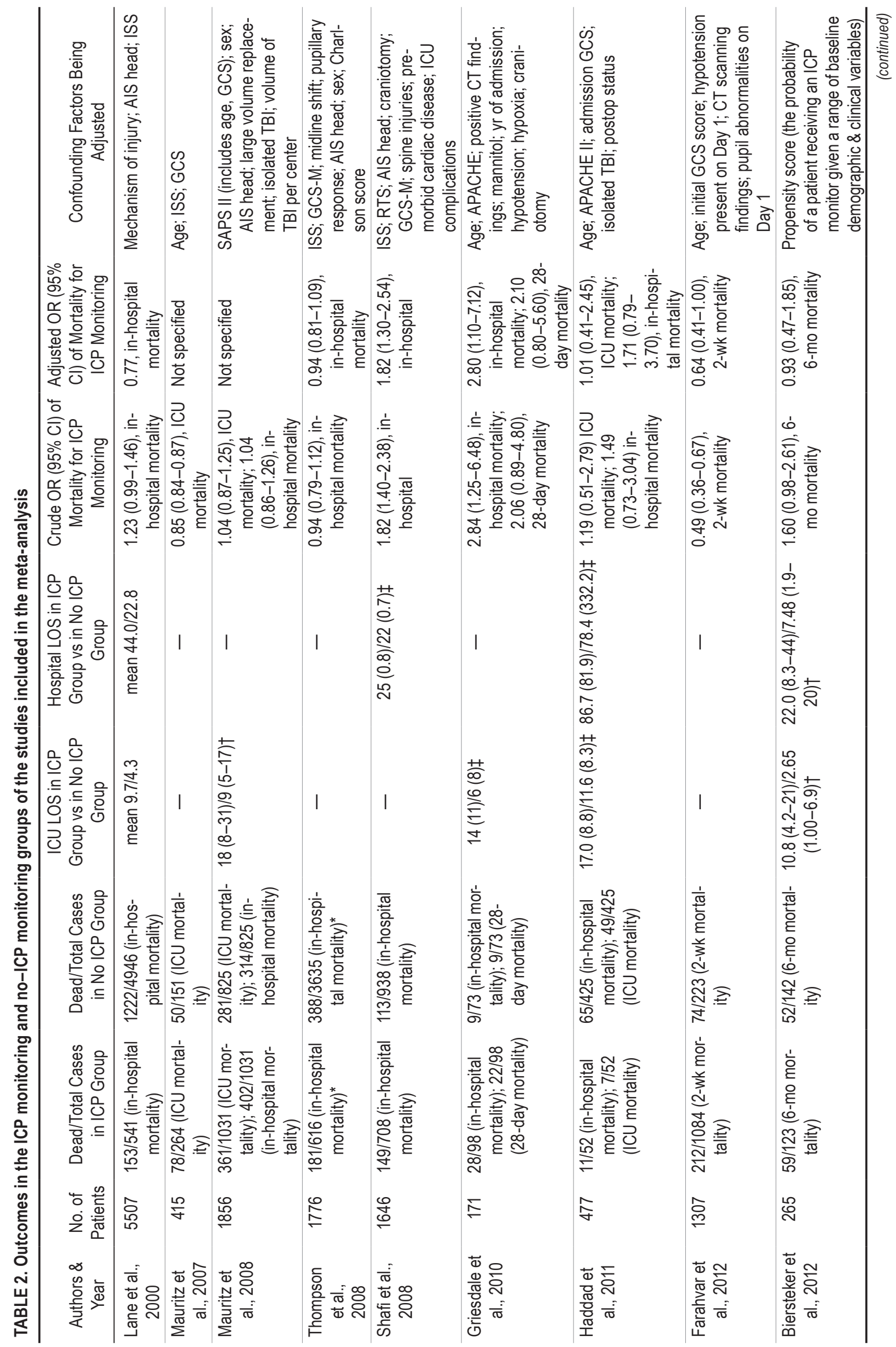




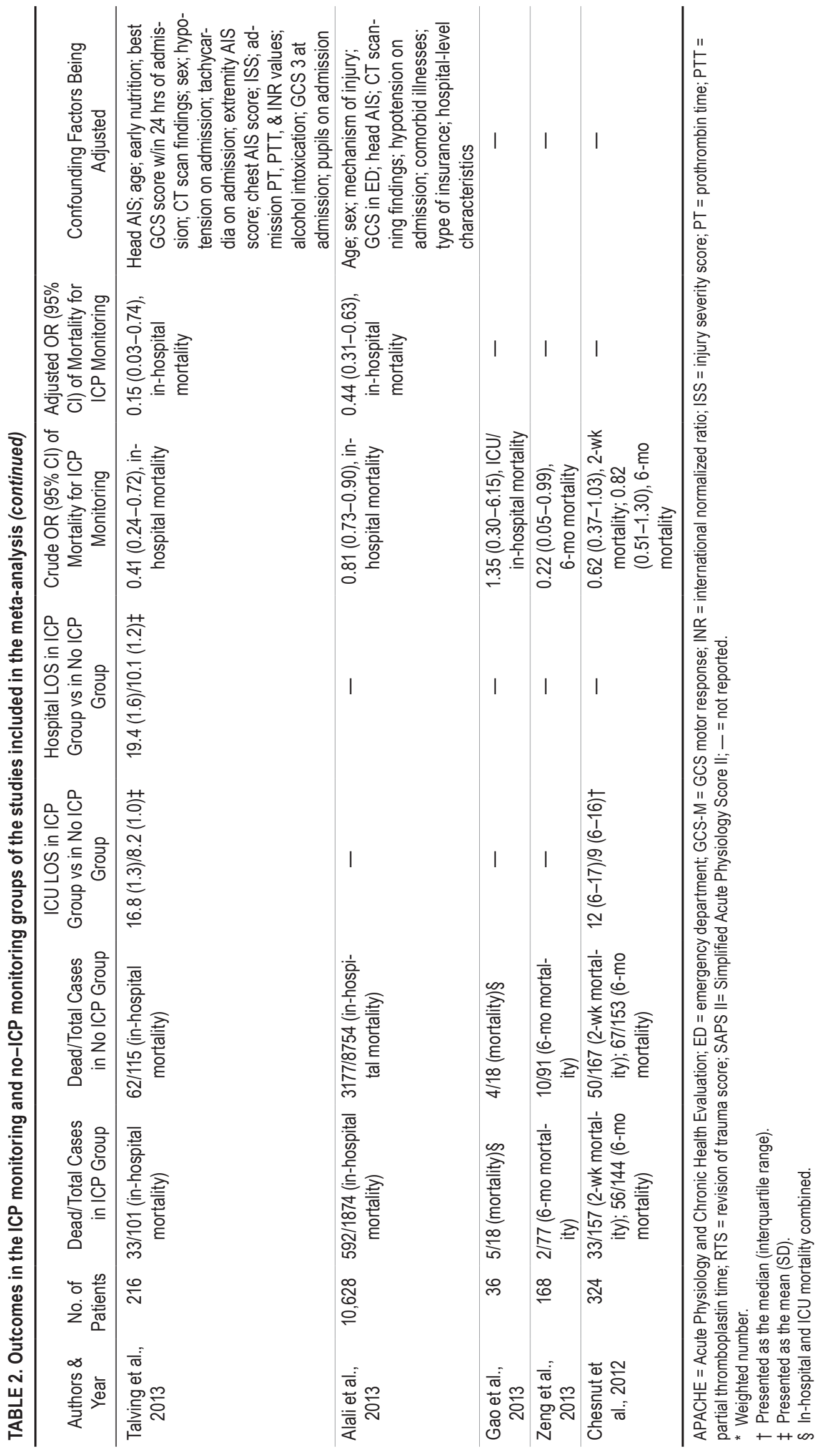




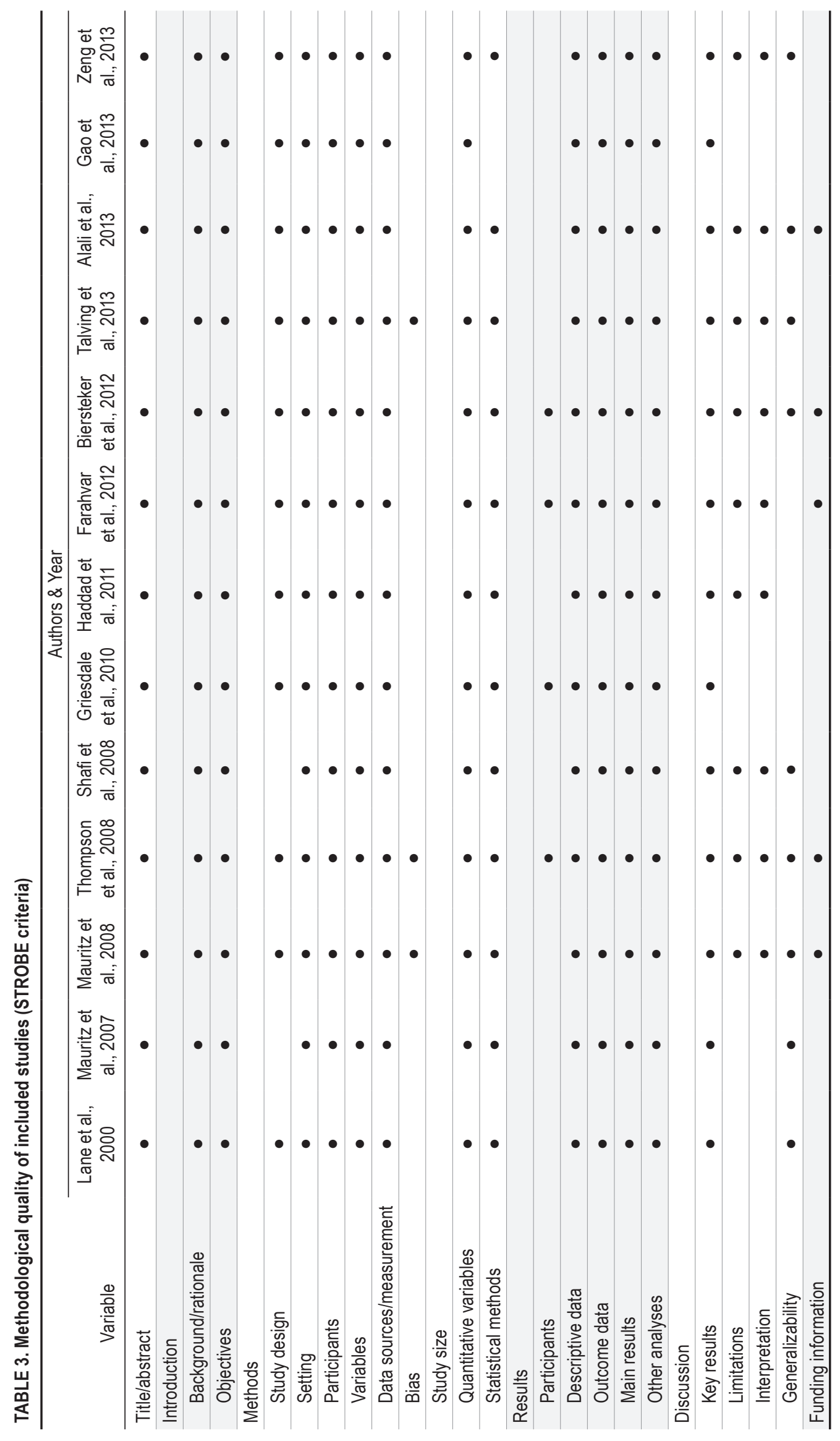


Odds Ratio

Odds Ratio

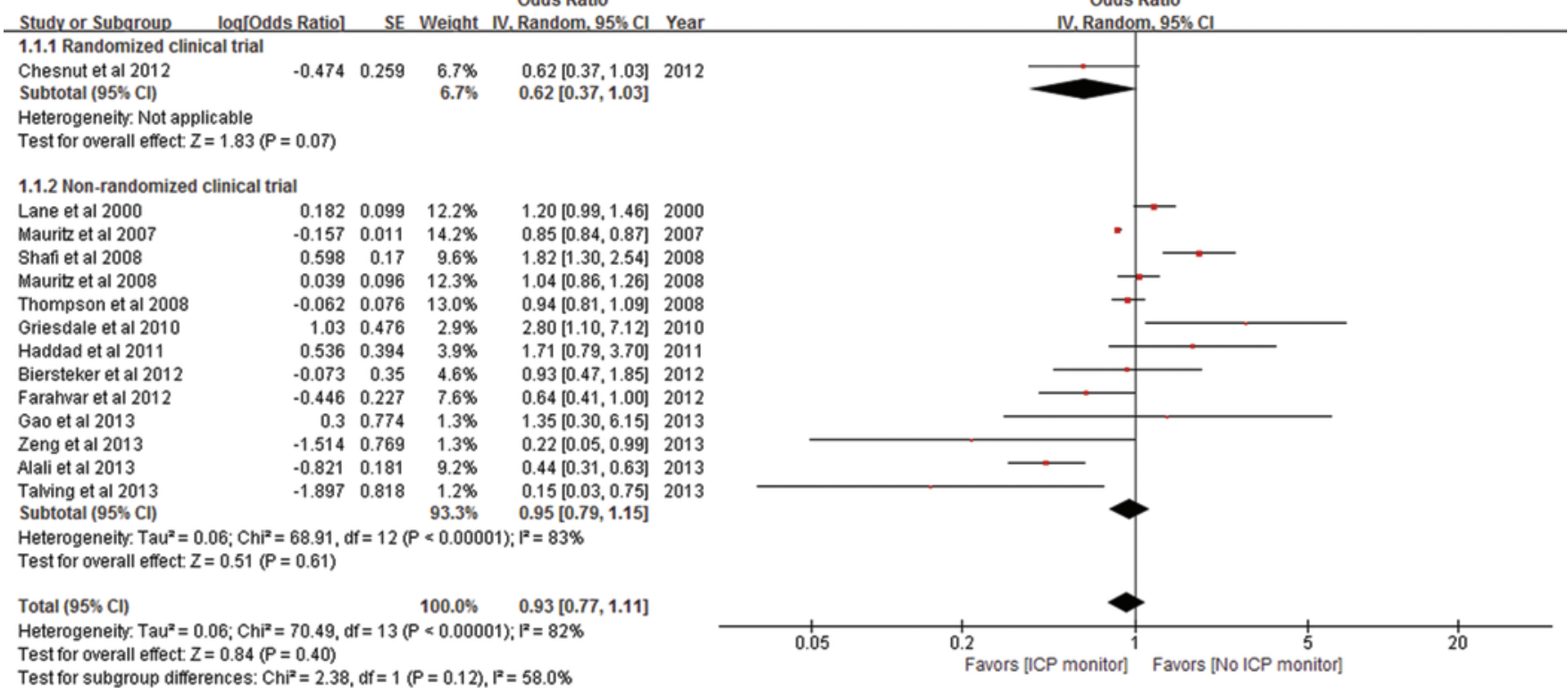

FIG. 2. Association between ICP monitoring and mortality in patients with TBI stratified by randomization or not (shown as $\log [$ Odds Ratio] with SE). IV = inverse variance. Figure is available in color online only.

a meta-analysis of clinical series on treatment for severe TBI since 1970 and found a $12 \%$ lower mortality rate and $6 \%$ more favorable outcome for patients who underwent aggressive ICP monitoring with intensive treatment; ${ }^{28}$ however, that meta-analysis concerned intensive treatment rather than ICP monitoring and not all patients who underwent intensive treatment underwent ICP monitoring. Also, the lack of a uniform definition of "intensive" or "aggressive" treatment is unfortunate.

This meta-analysis examined the association between ICP monitoring and outcome in patients with TBI. Fourteen studies compared mortality in TBI in an ICP monitor- ing group and a no-ICP monitoring group and reported no evidence that ICP monitoring decreased the odds of death. After excluding the only randomized controlled trial, the other 13 observational cohort studies evaluated the association between ICP monitoring and mortality in TBI patients and reported no evidence for a decreased risk of death with ICP monitoring. Although subgroup analysis revealed that ICP monitoring was significantly associated with a decrease in mortality versus no ICP monitoring in studies published after 2012, the isolated benefit of ICP monitoring in severe TBI is still not clearly established, because there is relatively little clinical evidence as to its

Odds Ratio

Odds Ratio

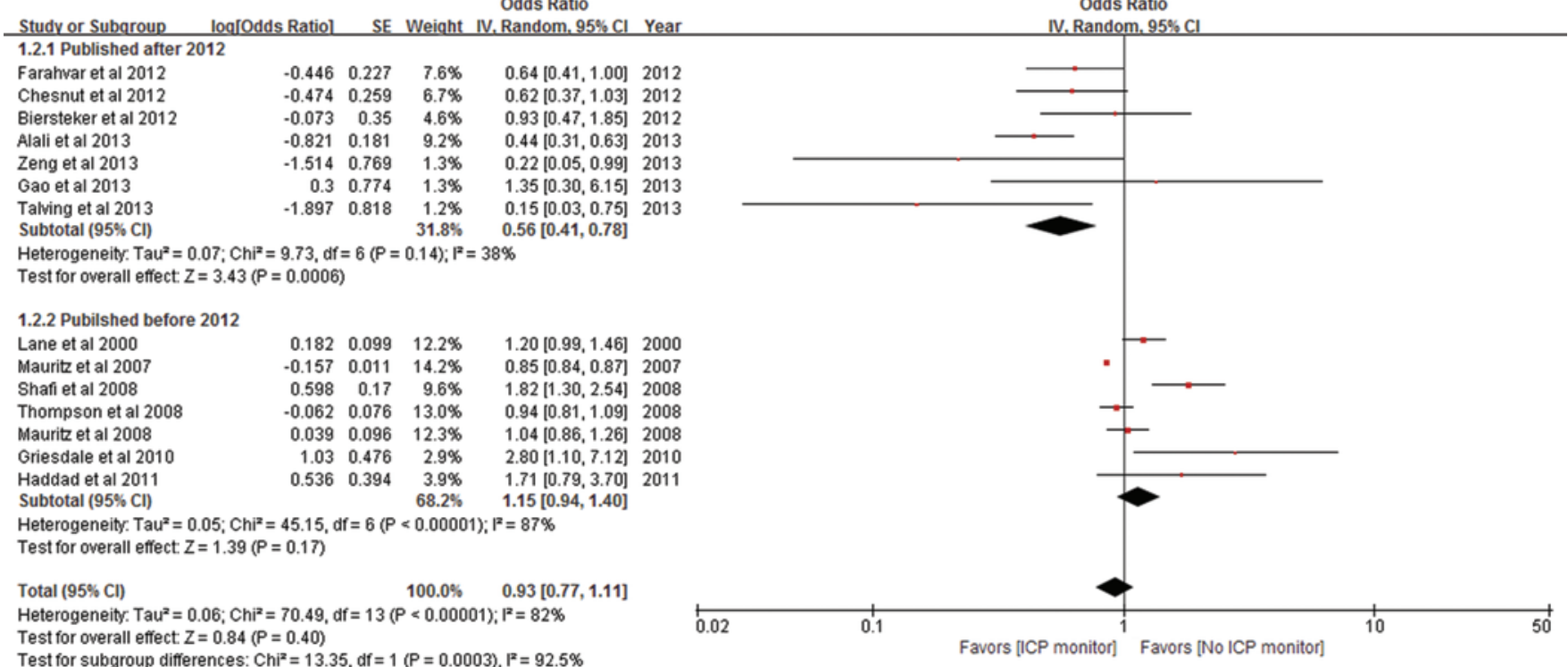

FIG. 3. Association between ICP monitoring and mortality in patients with TBI stratified by publication date (shown as log[Odds Ratio] with SE). Figure is available in color online only. 
Odds Ratio

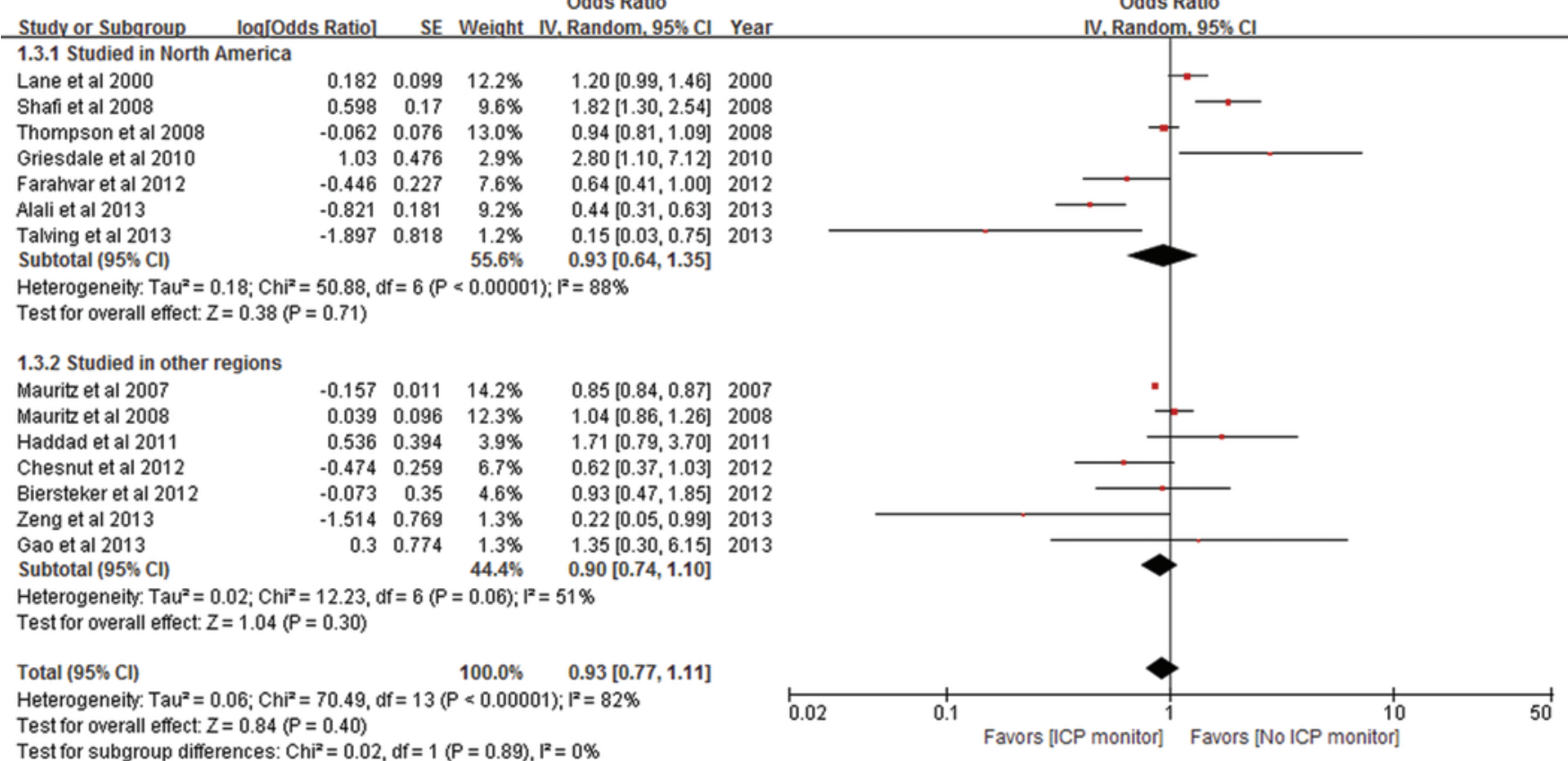

FIG. 4. Association between ICP monitoring and mortality in patients with TBI stratified by country (shown as log[Odds Ratio] with $\mathrm{SE})$. Figure is available in color online only.

efficacy. Additionally, pooled data analyses showed that both ICU LOS and hospital LOS with ICP monitoring were significantly longer than with no ICP monitoring.

There are several limitations to our meta-analysis. First, the major limitation was the relative dearth of studies that met our inclusion criteria. Second, all studies included in the meta-analysis were observational in nature, with the exception of one randomized controlled trial. Although most observational studies used multivariate analyses to determine an adjusted odds ratio after adjusting for many confounding factors, residual or unmeasured confounding remains a possible alternative explanation for our findings. Third, there was considerable heterogeneity for all the outcomes of interest. Heterogeneity among studies that assessed mortality was found by publication year and by the time period over which outcome was evaluated. Sensitivity

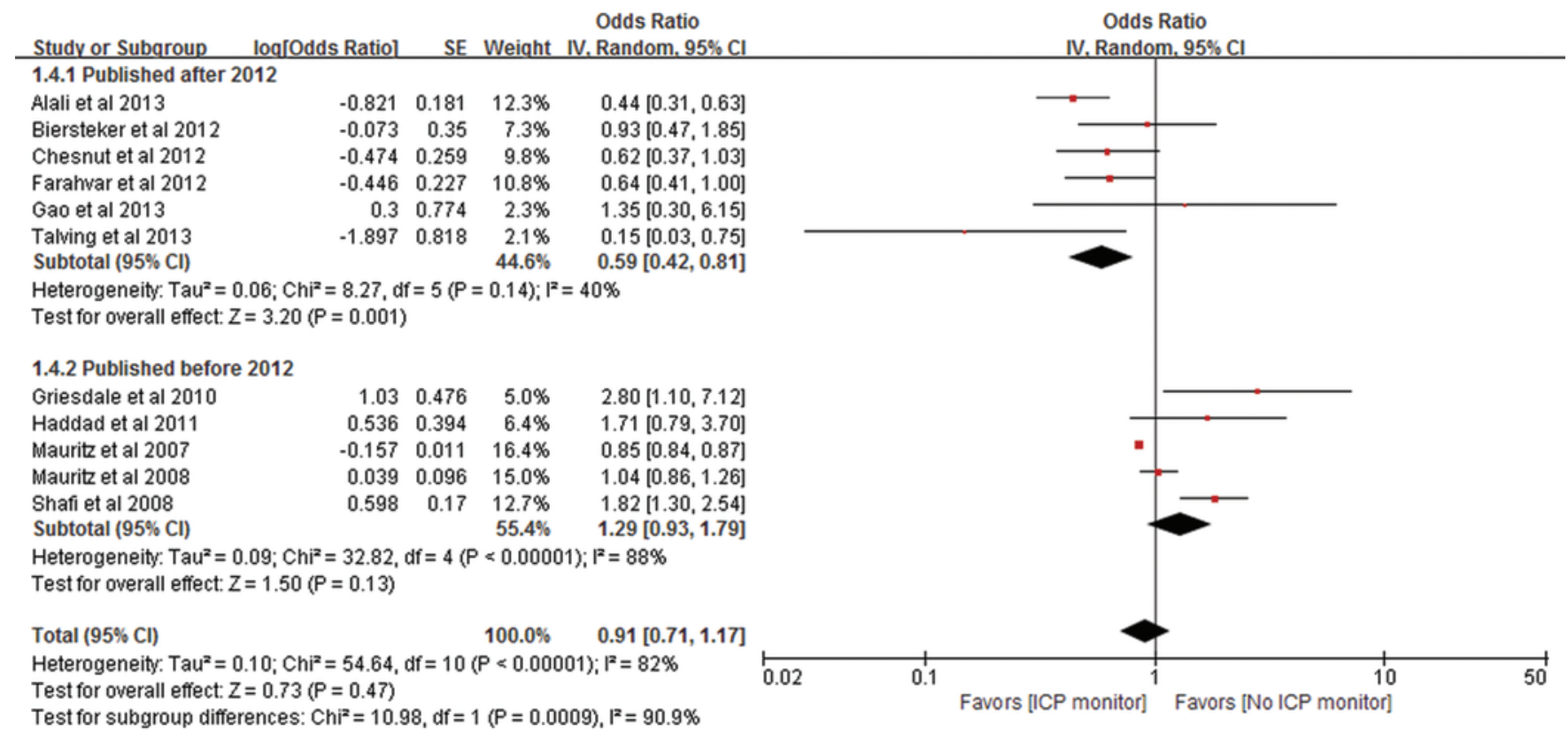

FIG. 5. Association between ICP monitoring and mortality in patients with severe TBI (GCS score < 9) stratified by publication date (shown as log[Odds Ratio] with SE). Figure is available in color online only. 


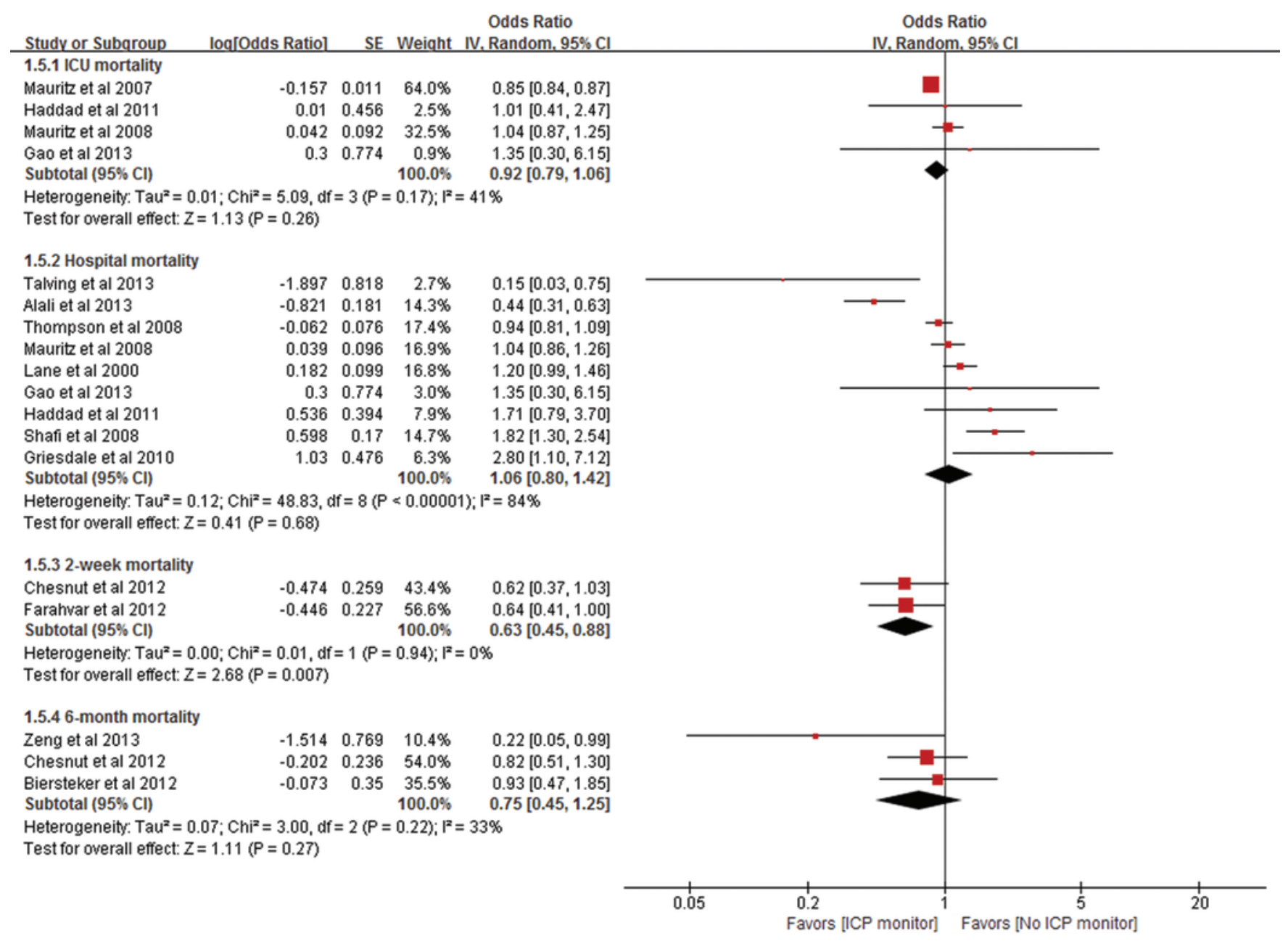

FIG. 6. Association between ICP monitoring and ICU mortality, hospital mortality, 2-week mortality, and 6-month mortality in patients with TBI (shown as log[Odds Ratio] with SE). Figure is available in color online only.

analyses, including country of origin, study design, sample size, and the timing of outcome evaluation after TBI, however, did not fully explain the observed heterogeneity for mortality. Finally, although we carried out our systematic review according to high methodological standards, the results of any meta-analysis are limited by the quality of the studies included. For example, only 4 studies presented a flow diagram for participants, indicating a risk of bias.

Our systematic review also had some important strengths. We conducted a thorough systematic search, included various databases, and used a comprehensive analytical method that allowed the inclusion of studies presenting not only crude odds ratios, but also adjusted odds ratios, thus enhancing the exhaustiveness of the results. Our rigorous methods were also based on established guidelines for conducting and reporting systematic reviews.

There are several potential reasons for the negative re-

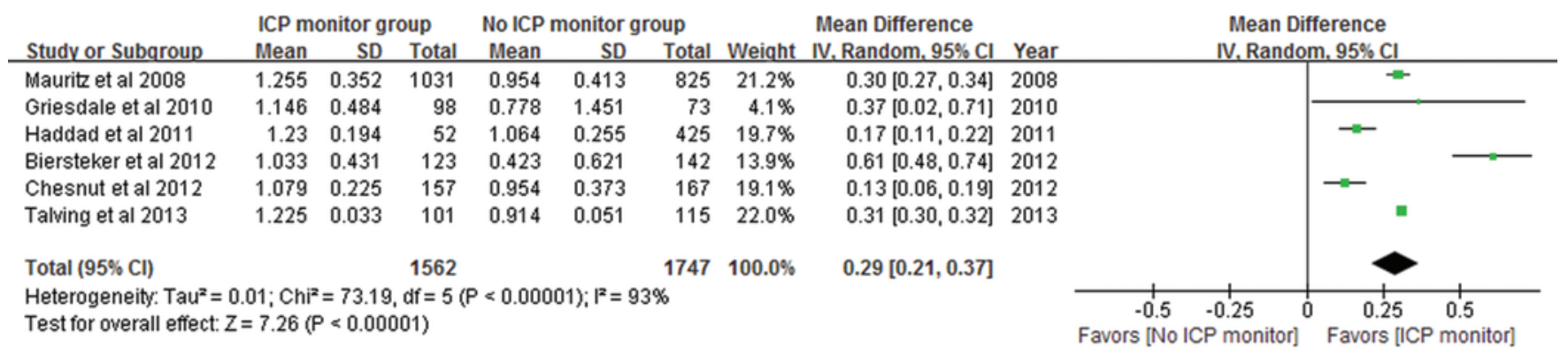

FIG. 7. Association between ICP monitoring and ICU LOS (shown as mean with SD in transformed days) in patients with TBI. Figure is available in color online only. 


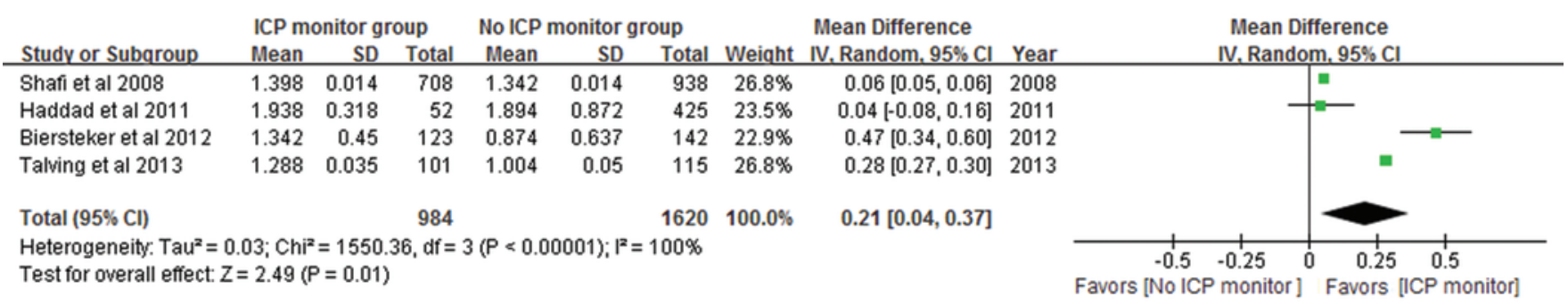

FIG. 8. Association between ICP monitoring and hospital LOS (shown as mean with SD in transformed days) in patients with TBI. Figure is available in color online only.

sults regarding ICP monitoring. First, because these studies were observational, with the exception of 1 randomized controlled trial, the decision to insert an ICP monitor had been made by the neurocritical care clinicians prior to the onset of the study. Thus, this decision may have been influenced by factors relating to the premorbid conditions of patients and the hospitals, the severity of the current TBI, and specific practices or preferences. Consequently, even with multivariate adjustment, determining an unbiased outcome estimate for ICP monitoring in these studies is problematic. This is exacerbated by the small sample sizes of some of the published studies, which limit adjustment for confounders.

Second, although the only randomized controlled trial included in the meta-analysis also reported that maintaining monitored ICP at $20 \mathrm{~mm} \mathrm{Hg}$ or lower in severe TBI patients was not superior to care based on imaging and clinical examination fidings, there was a limitation in the trial design. Specifically, the trial was conducted in South America, where practices in ICUs and aftercare may not be comparable with those in North America and Europe, and therefore lacks generalizability. Also, the intraparenchymal monitors used in the trial do not have the advantage of the external ventricular drains used in many ICUs, which allow drainage of spinal fluid to reduce pressure, and the primary outcome was not common mortality but a composite end point.7,25

Third, ICP monitoring would likely benefit only a subtype of TBI patients. Stratifying patients according to a multidimensional classification system of TBI is likely to

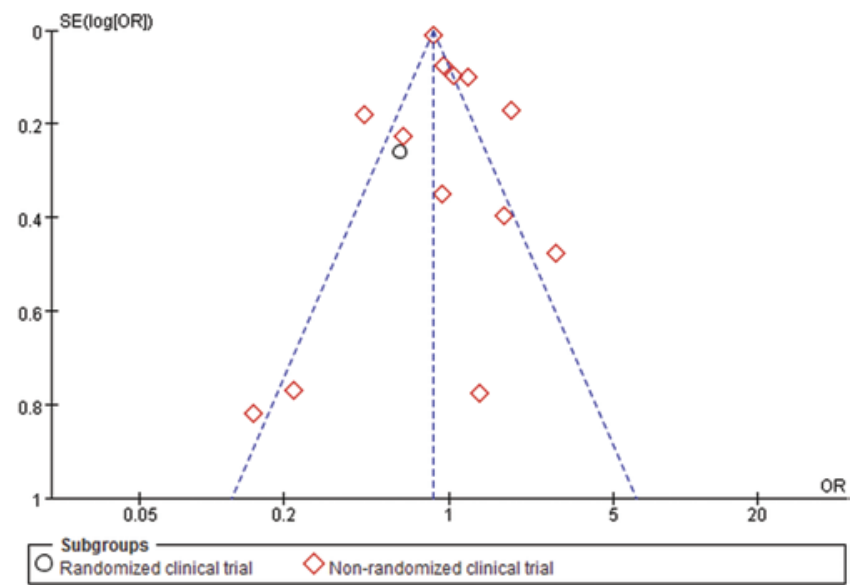

FIG. 9. Funnel plot for all studies included in the meta-analysis. Figure is available in color online only. be necessary to link specific patterns of brain injury with ICP monitoring and other types of evaluation that are most likely to be of benefit.

Fourth, the important difference between the information obtained from the ICP monitor and management based on this information must be highlighted. For example, a change in treatment can be instituted based on ICP or cerebral perfusion pressure findings. ${ }^{16}$ Moreover, ideal ICP and cerebral perfusion pressure target values have not been determined. Thus, clinicians may be able to gain indications and benefits not currently evident from use of ICP monitors.

Finally, patients who undergo ICP monitoring are subject to increased interventions based on information, such as brain tissue oxygen monitoring and central venous pressure, similar to other monitoring devices/technologies. ${ }^{20}$ We hope that interventions based on the information gained from these monitoring devices will improve outcomes. However, many of our current therapies for increased ICP, such as sedation and barbiturates, may be associated with worse outcomes in critically ill patients. ${ }^{11}$ Thus, lack of knowledge remains a confounding factor that cannot be ignored.

Subgroup analysis of the association between ICP monitoring and mortality in studies published after 2012 indicated a significant association between a decreased risk of death and ICP monitoring. This may be attributable to selection bias in recent years, but the more standardized TBI management and more rigorous compliance with BTF ICP monitoring guidelines in recent years, resulting in im-

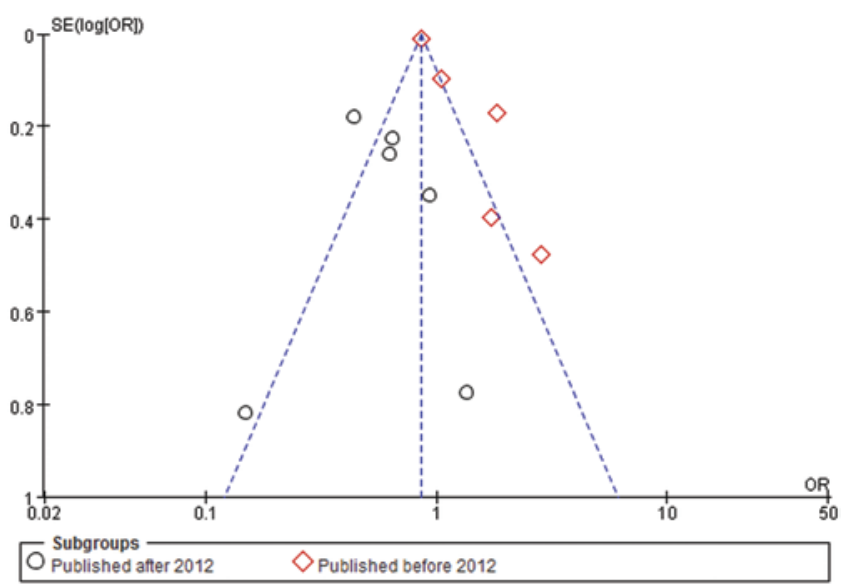

FIG. 10. Funnel plot for the studies including only severe TBI patients. Figure is available in color online only. 


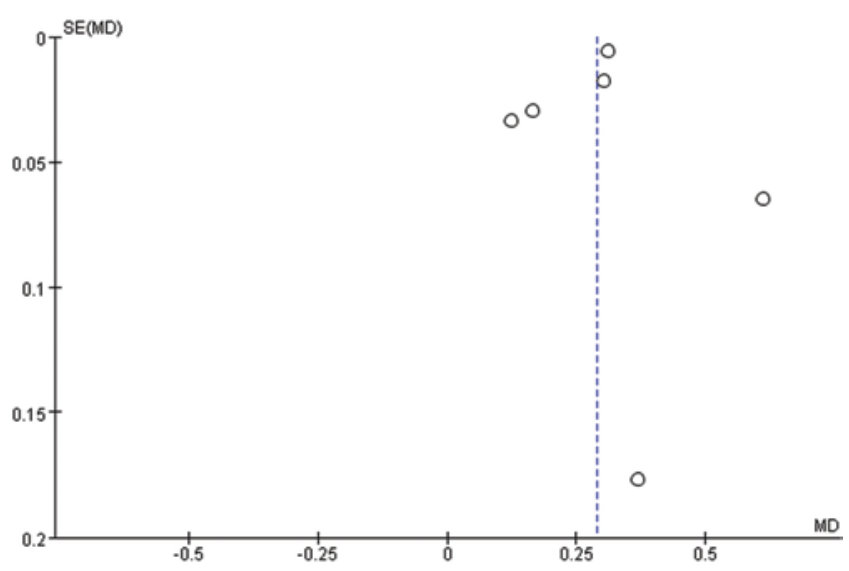

FIG. 11. Funnel plot for the studies of ICU LOS. Figure is available in color online only.

provements in TBI outcomes, may be a better alternative explanation. Several studies have assessed ICP monitoring utilization outcomes in TBI. Alali et al. stratified hospitals into quartiles based on ICP monitoring utilization and found that hospitals with higher rates of ICP monitoring use had lower mortality rates (OR 0.52 [95\% CI $0.35-$ 0.78])., ${ }^{1,30}$ Thus, ICP monitoring may influence outcomes independently, and thus further investigation is warranted.

Despite the fact that the BTF guidelines have some recommendations for ICP monitoring in patients with TBI, significant variability remains in the practice of ICP monitoring. Management plans based on ICP are controversial due to a lack of agreement in precise surgical and medical managements. This may be one reason for the significant heterogeneity in our study. Therefore, we first have to determine the optimal medical and surgical management plan and then randomize to ICP monitoring versus no ICP monitoring within a single management plan.

\section{Conclusions}

In this systematic review and meta-analysis of ICP monitoring studies including 24,792 patients, we found that the current clinical evidence does not indicate that ICP monitoring overall is significantly superior to no ICP mon-

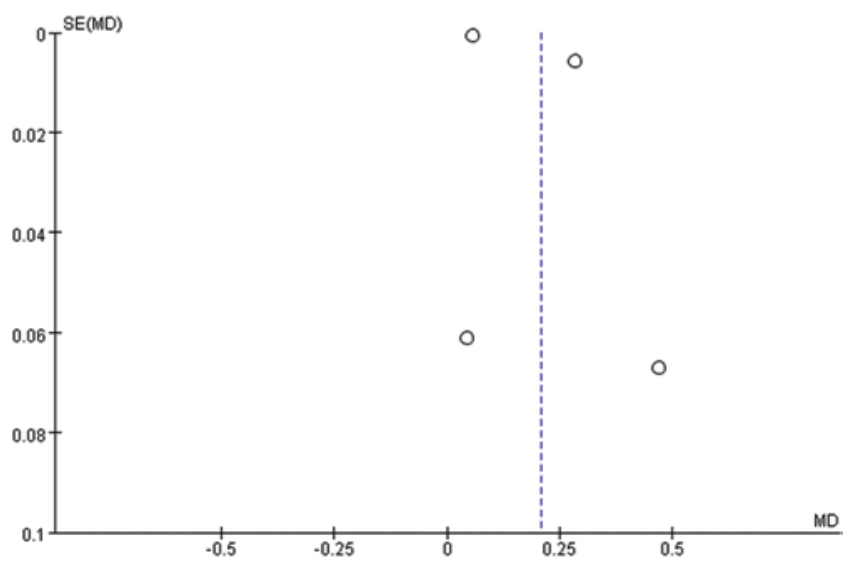

FIG. 12. Funnel plot for the studies of hospital LOS. Figure is available in color online only. itoring in terms of the mortality of TBI patients. However, studies published after 2012 indicated a lower mortality in patients who underwent ICP monitoring. Further effort should focus on standardizing ICP monitors and monitoring and on identifying optimal threshold values for ICPdirected care and distinct subgroups of severe TBI patients who may benefit from monitoring. This will improve the efficacy of ICP monitoring and help guide neurocritical care decisions in a shared decision-making process.

\section{References}

1. Alali AS, Fowler RA, Mainprize TG, Scales DC, Kiss A, de Mestral C, et al: Intracranial pressure monitoring in severe traumatic brain injury: results from the American College of Surgeons Trauma Quality Improvement Program. J Neurotrauma 30:1737-1746, 2013

2. Biersteker HA, Andriessen TM, Horn J, Franschman G, van der Naalt J, Hoedemaekers CW, et al: Factors influencing intracranial pressure monitoring guideline compliance and outcome after severe traumatic brain injury. Crit Care Med 40:1914-1922, 2012

3. Bland JM, Altman DG: The use of transformation when comparing two means. BMJ 312:1153, 1996

4. Bratton SL, Chestnut RM, Ghajar J, McConnell Hammond FF, Harris OA, Hartl R, et al: Guidelines for the management of severe traumatic brain injury. VIII. Intracranial pressure thresholds. J Neurotrauma 24 (Suppl 1):S55-S58, 2007 (Erratum in J Neurotrauma 25:276-278, 2008)

5. Bulger EM, Nathens AB, Rivara FP, Moore M, MacKenzie EJ, Jurkovich GJ: Management of severe head injury: institutional variations in care and effect on outcome. Crit Care Med 30:1870-1876, 2002

6. Bullock MR, Povlishock JT: Guidelines for the management of severe traumatic brain injury. Editor's commentary. J Neurotrauma 24 Suppl 1:vii-viii, 2007 (Erratum in J Neurotrauma $25: 276-278,2008$ )

7. Chesnut RM, Temkin N, Carney N, Dikmen S, Rondina C, Videtta W, et al: A trial of intracranial-pressure monitoring in traumatic brain injury. N Engl J Med 367:2471-2481, 2012

8. Farahvar A, Gerber LM, Chiu YL, Carney N, Härtl R, Ghajar $\mathrm{J}$ : Increased mortality in patients with severe traumatic brain injury treated without intracranial pressure monitoring. J Neurosurg 117:729-734, 2012

9. Forsyth RJ, Wolny S, Rodrigues B: Routine intracranial pressure monitoring in acute coma. Cochrane Database Syst $\operatorname{Rev}(2): C D 002043,2010$

10. Gao L, Wu X, Hu J, Jin Y, Han X, Wu X, et al: Intensive management and prognosis of 127 cases with traumatic bilateral frontal contusions. World Neurosurg 80:879-888, 2013

11. Girard TD, Kress JP, Fuchs BD, Thomason JW, Schweickert WD, Pun BT, et al: Efficacy and safety of a paired sedation and ventilator weaning protocol for mechanically ventilated patients in intensive care (Awakening and Breathing Controlled trial): a randomised controlled trial. Lancet 371:126134, 2008

12. Griesdale DE, McEwen J, Kurth T, Chittock DR: External ventricular drains and mortality in patients with severe traumatic brain injury. Can J Neurol Sci 37:43-48, 2010

13. Haddad S, Aldawood AS, Alferayan A, Russell NA, Tamim HM, Arabi YM: Relationship between intracranial pressure monitoring and outcomes in severe traumatic brain injury patients. Anaesth Intensive Care 39:1043-1050, 2011

14. Heneghan C: EBM resources on the new CEBM website. Evid Based Med 14:67, 2009

15. Higgins JP, Thompson SG, Deeks JJ, Altman DG: Measuring inconsistency in meta-analyses. BMJ 327:557-560, 2003 
16. Huang SJ, Hong WC, Han YY, Chen YS, Wen CS, Tsan YS, et al: Clinical outcome of severe head injury in different protocol-driven therapies. J Clin Neurosci 14:449-454, 2007

17. Hyder AA, Wunderlich CA, Puvanachandra P, Gururaj G, Kobusingye OC: The impact of traumatic brain injuries: a global perspective. NeuroRehabilitation 22:341-353, 2007

18. Juul N, Morris GF, Marshall SB, The Executive Committee of the International Selfotel Trial, Marshall LF: Intracranial hypertension and cerebral perfusion pressure: influence on neurological deterioration and outcome in severe head injury. J Neurosurg 92:1-6, 2000

19. Lane PL, Skoretz TG, Doig G, Girotti MJ: Intracranial pressure monitoring and outcomes after traumatic brain injury. Can J Surg 43:442-448, 2000

20. Martini RP, Deem S, Yanez ND, Chesnut RM, Weiss NS, Daniel S, et al: Management guided by brain tissue oxygen monitoring and outcome following severe traumatic brain injury. J Neurosurg 111:644-649, 2009

21. Mauritz W, Janciak I, Wilbacher I, Rusnak M: Severe traumatic brain injury in Austria IV: intensive care management. Wien Klin Wochenschr 119:46-55, 2007

22. Mauritz W, Steltzer H, Bauer P, Dolanski-Aghamanoukjan L, Metnitz P: Monitoring of intracranial pressure in patients with severe traumatic brain injury: an Austrian prospective multicenter study. Intensive Care Med 34:1208-1215, 2008

23. Murray GD, Butcher I, McHugh GS, Lu J, Mushkudiani NA, Maas AI, et al: Multivariable prognostic analysis in traumatic brain injury: results from the IMPACT study. J Neurotrauma 24:329-337, 2007

24. Roozenbeek B, Maas AI, Menon DK: Changing patterns in the epidemiology of traumatic brain injury. Nat Rev Neurol 9:231-236, 2013

25. Ropper AH: Brain in a box. N Engl J Med 367:2539-2541, 2012

26. Sahjpaul R, Girotti M: Intracranial pressure monitoring in severe traumatic brain injury-results of a Canadian survey. Can J Neurol Sci 27:143-147, 2000

27. Shafi S, Diaz-Arrastia R, Madden C, Gentilello L: Intracranial pressure monitoring in brain-injured patients is associated with worsening of survival. J Trauma 64:335-340, 2008

28. Stein SC, Georgoff P, Meghan S, Mirza KL, El Falaky OM:
Relationship of aggressive monitoring and treatment to improved outcomes in severe traumatic brain injury. J Neurosurg 112:1105-1112, 2010

29. Stocchetti N, Longhi L, Magnoni S, Roncati Zanier E, Canavesi K: Head injury, subarachnoid hemorrhage and intracranial pressure monitoring in Italy. Acta Neurochir (Wien) 145:761-765, 2003

30. Talving P, Karamanos E, Teixeira PG, Skiada D, Lam L, Belzberg H, et al: Intracranial pressure monitoring in severe head injury: compliance with Brain Trauma Foundation guidelines and effect on outcomes: a prospective study. $\mathbf{J}$ Neurosurg 119:1248-1254, 2013

31. Thompson HJ, Rivara FP, Jurkovich GJ, Wang J, Nathens $\mathrm{AB}$, MacKenzie EJ: Evaluation of the effect of intensity of care on mortality after traumatic brain injury. Crit Care Med 36:282-290, 2008

32. von Elm E, Altman DG, Egger M, Pocock SJ, Gøtzsche PC, Vandenbroucke JP: The Strengthening the Reporting of Observational Studies in Epidemiology (STROBE) statement: guidelines for reporting observational studies. Lancet 370:1453-1457, 2007

33. Zeng J, Tong W, Zheng P: Decreased risk of acute kidney injury with intracranial pressure monitoring in patients with moderate or severe brain injury. J Neurosurg 119:12281232,2013

\section{Author Contributions}

Conception and design: Hu. Acquisition of data: Yuan, Wu. Analysis and interpretation of data: Yuan, Wu, Sun. Drafting the article: Yuan, Wu, Sun. Critically revising the article: all authors. Reviewed submitted version of manuscript: all authors. Approved the final version of the manuscript on behalf of all authors: $\mathrm{Hu}$. Statistical analysis: Yuan, Wu. Study supervision: Hu.

\section{Correspondence}

Jin $\mathrm{Hu}$, Department of Neurosurgery, Huashan Hospital, Fudan University, 12 Wulumuqi Zhong Rd., Shanghai 200040, China. email: 297260090@qq.com. 